\title{
SPL Document Type Terminology
}

National Cancer Institute

\section{Source}

National Cancer Institute. SPL Document Type Terminology. NCI Thesaurus. Code C87299.

Terminology that represents kinds of documents in supporting regulatory submissions in electronic format - drug establishment registration and drug listing. 